\title{
Does Percutaneous Lumbosacral Pedicle Screw Instrumentation Prevent Long-Term Adjacent Segment Disease after Lumbar Fusion?
}

\author{
Stuart Changoor, Michael Joseph Faloon, Conor John Dunn, Nikhil Sahai, \\ Kimona Issa, Kumar Sinha, Ki Soo Hwang, Arash Emami \\ Deparment of Orthopaedic Surgery, St. Joseph's Regional Medical Center, Paterson, NJ, USA
}

\begin{abstract}
Study Design: Retrospective cohort study.
Purpose: To assess long-term clinical outcomes of adjacent segment disease (ASD) in patients who underwent lumbar interbody fusion with percutaneous pedicle screw (PS) instrumentation.

Overview of Literature: ASD is a well-known sequela of spinal fusion, and is reported to occur at a rate of $2 \%-3 \%$ per year. There is debate as to whether ASD is a result of the instrumentation and fusion method or is the natural history of the patient's disease. Minimally invasive percutaneous PS augmentation of lumbar interbody fusion aims to prevent the disruption of posterior soft tissue stabilizers.

Methods: From 2004-2014, 419 consecutive patients underwent anterior, lateral, or minimally invasive transforaminal lumbar interbody fusion with percutaneous PS placement at a single institution. The mean follow-up was 4.5 years. The primary outcome measure was reoperation due to ASD. Patients were divided into two cohorts: those who underwent revision surgery secondary to ASD and those who did not require further surgery. Radiographic parameters were performed using postoperative radiographs. Patients with a pelvic incidence-lumbar lordosis (PI-LL) mismatch $>10^{\circ}$ were noted.

Results: Revision proportion secondary to ASD was $4.77 \%$ ( $n=20)$. Mean time to revision surgery was 2.5 years. Revision rate secondary to ASD was $1.1 \%$ per year. Patients who developed ASD were younger than those who did not ( 50.5 vs. 56.9 years, $p=0.015)$. There was no difference in number of levels fused between cohorts. Revision proportion secondary to ASD was similar between approaches (anterior, lateral, minimally invasive). There was no significant difference in PI-LL mismatch between those who underwent revision for $A S D$ and those who did not (22.2\% vs. $18.8 \%, p=0.758)$.

Conclusions: ASD rates in patients who underwent percutaneous PS placement were lower than those previously published after open PS placement, possibly related to greater preservation of the posterior stabilizing elements of the lumbar spine.
\end{abstract}

Keywords: Lumbar fusion; Adjacent segment disease; Revision; Minimally invasive; Percutaneous; Pedicle screws; Complications; Pelvic parameters

Received Apr 7, 2020; Accepted Apr 27, 2020

Corresponding author: Michael Faloon

University Spine Center, 504 Valley Road, Suite 203, Wayne, NJ 07470, USA

Tel: +1-973-686-0700 (x199), Fax: +1-973-686-0701, E-mail: faloonresearch@gmail.com; faloonm@sjhmc.org 


\section{Introduction}

Adjacent segment disease (ASD) of the lumbar spine is a common sequela of lumbar spinal fusion procedures, which is reported to occur at a rate of $2 \%-3 \%$ per year following operative stabilization [1]. Reducing the rate of ASD in these patients is of great concern to spine surgeons and the healthcare system, given revision operations add substantial costs to the treatment of common pathologies of the lumbar spine and can result in higher complications and poorer functional outcomes $[2,3]$.

The nature of the development of ASD after lumbar fusion is currently debated in the literature. Some authors suggest that ASD is due to the natural history of the patient's condition, whereas others suggest that the instrumentation and method of fusion impact the rate of ASD $[1,4,5]$. Minimally invasive percutaneous lumbosacral pedicle screw instrumentation has been suggested to reduce the incidence of ASD by preventing the disruption of posterior soft tissue stabilizers and paraspinal muscle dissection, as well as reducing iatrogenic injury to the proximal facet joint [6,7]. Additionally, abnormal sagittal plane configuration of the spine following lumbar fusion has been hypothesized to increase the rate of ASD $[8,9]$.

In theory, the rate of ASD requiring revision surgery could be lower in patients who undergo fusion procedures with percutaneous pedicle screw instrumentation than in those who undergo traditional lumbar fusion procedures with open pedicle screw augmentation. Few studies in the literature have addressed this hypothesis, which is of great importance to the management of ubiquitous spinal pathology. Therefore, the purpose of this study was to (1) describe the rate of revision surgery secondary to ASD in patients who underwent lumbar fusion procedures with minimally invasive percutaneous lumbosacral pedicle screw instrumentation; and (2) to asses risk factors for the development of ASD, including demographics, surgical approach, and pelvic incidence-lumbar lordosis (PI-LL) mismatch in these patients.

\section{Materials and Methods}

Institutional review board approval was obtained for this study from the institutional review board of St. Joseph's University Medical Center (approval no., PR\#08-034). A retrospective review of the database of St. Joseph's Univerity Medical Center from 2004 to 2014 was performed to identify patients who underwent anterior lumbar interbody fusion (ALIF), lateral lumbar interbody fusion (LLIF), or minimally-invasive transforaminal lumbar interbody fusion (mis-TLIF) with minimally invasive percutaneous lumbosacral pedicle screw instrumentation augmentation. The initial search resulted in 456 consecutive patients. Patients with a minimum follow-up of 2 years were included in the study, with 37 patients lost to follow-up before this threshold.

The primary outcome measure was reoperation due to ASD refractory to further conservative interventions. ASD was defined as the appearance of new clinical symptoms, attributable to adjacent segment degeneration, diagnosed by a fellowship-trained orthopedic spine surgeon, which required or was indicated for surgical revision.

Patients were divided into two groups: those who underwent revision surgery secondary to ASD and those who did not require revision surgery after the index procedure. Demographic information including age, sex, body mass index (BMI), number of levels fused, and smoking status were recorded and compared between the two groups. Smokers were defined as those who were active tobacco users or those who had quit within 6 weeks prior to surgery. Information regarding risk factor analysis in the form of American Society of Anesthesiologists Physical Status Classification System grading (ASA grade) was also recorded and compared between the groups. Surgical approach (ALIF, LLIF, mis-TLIF), primary operative diagnosis for the index procedure, and reoperations were noted. All surgical procedures were performed by fellowship-trained orthopedic spine surgeons.

Radiographic evaluation, including lumbopelvic parameter measurements, were performed for all patients, using immediate postoperative standing sagittal lumbar radiographs. PI-LL mismatch, defined as a difference between PI and LL greater than $10^{\circ}$, was calculated. The percentage of PI-LL mismatch was compared between the two groups.

All outcome metrics were tabulated in an Excel spreadsheet (Microsoft Corp., Redmond, WA, USA) for statistical analysis. A Kaplan-Meier survivorship curve was constructed to demonstrate the expected proportion of ASD per year of follow-up. The statistical software Graph Pad Prism ver. 5.01 (GraphPad Software Inc., La Jolla, CA, USA), was used for all statistical calculations. A statistical analysis was performed, including Student $t$-test for means and Fisher's exact test for categorical variables, and $p$-values were calculated. A $p$-value of 0.05 was considered statistically significant. 


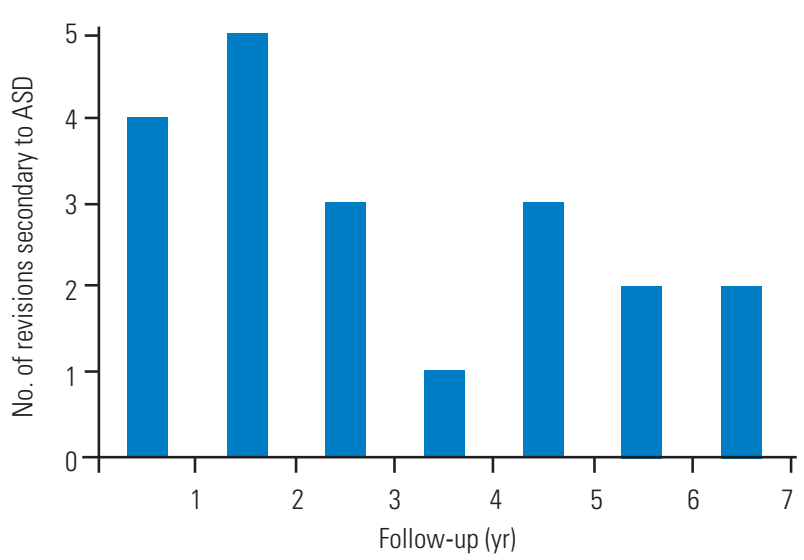

Fig. 1. Revisions per year secondary to ASD. The highest number of revisions occurred during the second year of postoperative follow-up. ASD, adjacent segment disease.

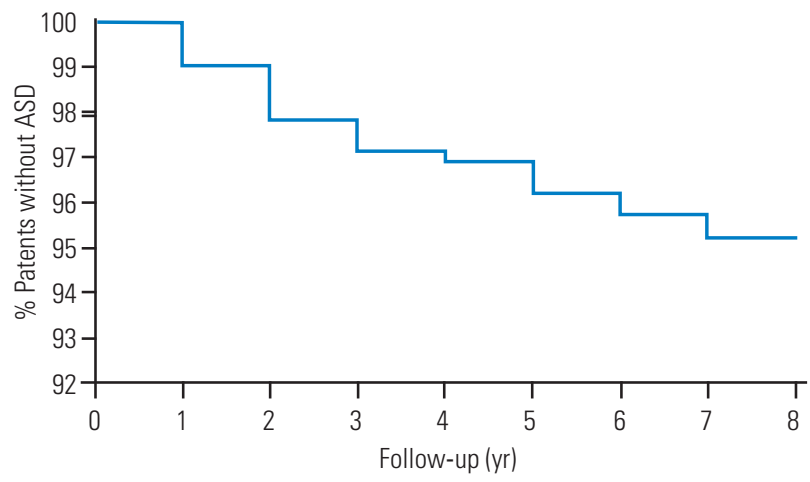

Fig. 2. Kaplan-Meier survivorship curve. Each point represents the percentage of patients who were expected to remain without ASD for each year of followup. ASD, adjacent segment disease.

\section{Results}

A total of 419 patients were included in the final analysis, with a mean follow-up of 4.5 years (range, 3-12 years). Twenty patients (4.8\%) underwent revision surgery for symptomatic ASD, indicating a $1.1 \%$ revision rate for ASD per year. Of these patients, the mean time to revision surgery was 2.5 years (range, $0.5-6.8$ years). The number of revisions secondary to ASD varied during each year of follow-up, with the highest number during the second year of postoperative follow-up at five revisions (Fig. 1). The Kaplan-Meier survivorship analysis predicted a disease-free survival rate of $96.2 \%$ at 5 years after the index fusion procedure (Fig. 2).

A total of 185 men and 234 women were included in the analysis, with a mean age of 56 years (range, 23-76 years), BMI of $28.9 \mathrm{~kg} / \mathrm{m}^{2}$ (range, $21.4-33.8 \mathrm{~kg} / \mathrm{m}^{2}$ ), ASA grade of 1.9 (range, 1-3), 36 of whom were smokers. The average number of spinal levels fused was $1.4 \pm 0.3$ levels. Patients who required revision secondary to ASD were younger than those who did not, with a mean age of $50.5 \pm 12.5$ years versus $56.9 \pm 11.5$ years $(p=0.015)$. There was no difference in the number of spinal levels initially fused between patients who underwent revision surgery for ASD and those who did not (1.6 versus 1.4 levels, $p=0.248$ ). No other demographic factors reached statistical significance (Table 1). There was no difference between the two cohorts in terms of primary operative indication or surgical approach and fusion technique. Similar proportions of revisions secondary to ASD were observed between patients who underwent ALIF, mis-TLIF, and LLIF procedures supplemented with percutaneous pedicle screws (Table

Table 1. Patient demographics overall and between cohorts

\begin{tabular}{|c|c|c|c|c|}
\hline Characteristic & Overall & No revision ASD & Revision ASD & $p$-value \\
\hline No. of patients & 419 & 399 & 20 & \\
\hline Age (yr) & $55.9(23-76)$ & $56.9(35-76)$ & $50.5(23-68)$ & 0.015 \\
\hline Sex & & & & 1.000 \\
\hline Men & 185 & 176 & 9 & \\
\hline Women & 234 & 223 & 11 & \\
\hline Body mass index $\left(\mathrm{kg} / \mathrm{m}^{2}\right)$ & 28.9 & 28.6 & 29.2 & 0.475 \\
\hline American Society of Anesthesiologists grade & $1.9(1-3)$ & $1.9(1-3)$ & $2.1(1-3)$ & 0.329 \\
\hline Smoking history (yes) & $36(8.6)$ & $34(8.5)$ & $2(10)$ & 0.686 \\
\hline No. of levels fused & $1.4(1-3)$ & $1.4(1-3)$ & $1.6(1-3)$ & 0.248 \\
\hline
\end{tabular}

Values are presented as number, mean (range), or number (\%).

ASD, adjacent segment disease. 
Table 2. Surgical indications and fusion techniques overall and between cohorts

\begin{tabular}{|c|c|c|c|c|}
\hline Variable & Overall & No revision ASD & Revision ASD & $p$-value \\
\hline \multicolumn{5}{|l|}{ Primary operative indication } \\
\hline Degenerative disc disease & $190(42.1)$ & $180(94.7)$ & $10(5.3)$ & 0.819 \\
\hline Spondylolisthesis & $68(15.1)$ & $65(95.6)$ & $3(4.4)$ & 1.000 \\
\hline Post laminectomy syndrome/recurrent herniated disc & $161(35.7)$ & $154(95.7)$ & $7(4.3)$ & 0.817 \\
\hline \multicolumn{5}{|l|}{ Surgical approach to fusion } \\
\hline Anterior lumbar interbody fusion & $195(43.2)$ & $187(95.9)$ & $8(4.1)$ & 0.649 \\
\hline Minimally-invasive transforaminal lumbar interbody fusion & $164(36.4)$ & $156(95.1)$ & $8(4.9)$ & 1.000 \\
\hline Lateral lumbar interbody fusion & $60(13.3)$ & $56(93.3)$ & $4(6.7)$ & 0.508 \\
\hline
\end{tabular}

Values are presented as number (\%).

ASD, adjacent segment disease.

2). Of the patients who were revised, nine had a herniated disc, seven had stenosis, and four had disc degeneration with retrolisthesis.

Four $(20 \%)$ of the 20 patients who underwent revision surgery for symptomatic ASD had a PI-LL mismatch $>10^{\circ}$. Of the 399 patients who did not require revision surgery secondary to ASD, 71 patients (17.8\%) were found to have an observed difference of PI from LL of $>10^{\circ}$. The difference in proportion of PI-LL mismatch between cohorts was not statistically significant $(p=0.767$ ).

The reoperation proportion of the overall cohort for any complication was $10.9 \%(n=46)$. In addition to the 20 patients who underwent revision surgery for symptomatic ASD, 16 patients were revised for symptomatic pseudarthrosis, seven patients had screws revised due to confirmed misplacement with clinical symptomatology, one patient returned to the operating room after rod disengagement, one patient was revised for instrumentation failure and prominence, and one patient had a surgical site infection treated with surgical washout and intravenous antibiotics.

\section{Discussion}

The effect of various spinal interventions and their effect on the continued degenerative changes of the lumbar spine remains unclear, specifically in regard to adjacent segment degeneration and disease [1]. The conclusions of various studies conflict in terms of the degree of effect that various lumbosacral interventions have on the rate of ASD, a common and debilitating complication [10,11]. Although some authors claim that the natural history of spine degeneration dictates the majority of revision cases secondary to ASD, others have deduced that the specific procedure, instrumentation, and certain risk factors are attributable to an increased rate of ASD $[1,4,12,13]$. It has been suggested that reduced destruction of the posterior stabilizers of the lumbar spine characterized by minimally invasive instrumentation and fusion could reduce the rate of ASD and thus the need for costly revision surgeries [1418]. Conversely, others suggest that use of the percutaneous method increases the incidence of superior articular facet violation, theoretically impacting ASD incidence [19]. However, the proper technique should preclude this complication. Nevertheless, there are few reports addressing the rate of ASD in these minimally invasive lumbosacral spinal fusions. Therefore, the purpose of this study was to establish a rate of ASD in the literature, defined by necessary revision surgery for clinical symptoms attributable to adjacent segment degeneration, in lumbosacral spinal fusions using a percutaneous technique for pedicle screw placement.

Several small studies have shown that minimally-invasive approaches to lumbosacral fusion can reduce the rate of revision due to ASD. In a study with 52 patients (mean age, $50 \pm 14$ years) who underwent mis-TLIF, Yee et al. [2] had demonstrated a $7.69 \%$ rate of ASD compared with $18.75 \%$ in patients treated via the open technique. The open cohort only contained 16 patients, and the overall mean radiographic follow-up was $33 \pm 20$ months. Their mean time to ASD diagnosis was $48 \pm 18$ months. Their MIS-TLIF population was significantly younger and still maintained a lower rate of ASD. Contrary to this finding, the analysis of our population found that younger patients were more likely to develop ASD.

In a study by Parker et al. [20], patients who underwent 
mis-TLIF had a significantly reduced rate of ASD than those who underwent open TLIF at 5 years follow-up (9.3\% compared to $22.7 \%$ ). In a matched pair comparison study by Seng et al. [21] consisting of 40 patients with a mean age of $56.7 \pm 1.65$ years, the authors found the same rate of ASD in both the mis-TLIF and open TLIF cohorts (10\%) while demonstrating all of the well-described advantages of minimally invasive surgery, such as less blood loss and narcotic usage, earlier ambulation, and shorter hospitalizations. The cohorts were matched in age, BMI, smoking status, spinal level, disease etiology, and number of major comorbidities. They also evaluated functional clinical outcomes, which revealed similar outcomes between both open and mis-TLIF cohorts at 5 years' postoperative follow-up. Furthermore, Radcliff et al. [4] had found a $30 \%$ rate of ASD (including nonsurgical treatment modalities) in both minimally invasive and open cohorts for patients who had undergone instrumented one- or two-level posterior lumbar fusions in 58 patients with 3.8 years' follow-up.

The reported rate of ASD following instrumented lumbar fusion varies substantially in the literature and increases at a steady rate as the length of follow-up increases, reaching as high as $30.3 \%$ in one study with pedicle screw instrumentation and a 5-year follow-up [22]. We defined ASD as patients who required revision surgery, which partially explains why our rate of $4.77 \%$ at a mean follow-up of 4.5 years is comparatively lower than some in the literature. This percentage was still substantially lower than the $16.5 \%$ of patients requiring revision due to ASD observed by Ghiselli et al. [23].

In a systematic review conducted by Radcliff et al. [1] in 2013, the overall reported rate of ASD after any decompression and stabilization procedure was found to be approximately $2 \%$ to $3 \%$ per patient per year. They also found that laminectomy adjacent to a fusion and sagittal imbalance were consistently associated with the development of ASD [1]. In the current study, we observed an ASD rate of $1.1 \%$ per year after lumbar fusion augmented with percutaneous pedicle screw instrumentation. Given the increasing nature of ASD over time, future studies must be carried out to 10 years, especially in younger patients, to fully appreciate the effect of ASD by chosen intervention. Our findings, at 4.5 years (range, 3-12 years), suggest that greater preservation of the posterior stabilizing elements of the lumbar spine with the percutaneous technique plays a significant role in decreasing the overall rate of revision due to ASD at lengthy follow-up intervals.

In a 2005 study with 112 patients and a mean followup of 7 years, Aiki et al. [24] had found that multilevel fusions were associated with an increased risk of revision overall in a case series of patients undergoing open posterior lumbar fusions. They also reported an overall revision rate secondary to ASD of $7.7 \%$. Our study found no significant relationship between revisions due to ASD and levels fused ( 1.6 versus $1.4, p=0.208$ ). However, the analysis of our population suggested that patients requiring a revision procedure due to ASD were significantly younger at the time of the index operation than those who did not develop ASD (50.5 versus 56.9 years, $p=0.015$ ).

Kumar et al. [8] had concluded that postoperative sagittal alignment is an important driving factor in the development of adjacent segment degeneration. Several studies have further demonstrated the strength of this correlation, specifically the mismatch between PI and lumbar lordosis, with a difference of $\pm 10^{\circ}$ considered optimal $[9,25]$. Accordingly, our study also found a higher percentage of PI and LL mismatch with greater than $10^{\circ}$ of difference in the ASD cohort (22.2\% versus $18.8 \%$ ); however, we did not find this observation to be statistically significant ( $p=0.758)$.

Our study was limited by several factors. The retrospective nature of the study could have introduced selection bias. Furthermore, we did not have a comparison group with patients who underwent fusion procedures with open pedicle screw instrumentation. Nonetheless, the power of the study remains a strength, given no other clinical case series has examined a large series of patients undergoing a homogenous percutaneous technique with such a long-term follow-up. Further studies should follow up patients undergoing similar minimally invasive lumbosacral fusions up to 10 years to more accurately assess the rate of ASD and revision due to ASD along with clinical function scores, to further investigate the efficacy of this technique.

\section{Conclusions}

In conclusion, percutaneous lumbosacral pedicle screw instrumentation might reduce the incidence of long-term revisions due to ASD, as demonstrated by our population's revision proportion of $4.77 \%$, revision rate of $1.1 \%$ per year, and 4.5-year mean follow-up. Although the natural history of ASD might not be fully prevented with any 
surgical intervention yet described, reducing its incidence is of primary concern to the patient, spine surgeon, and health care system at large due to its abundant cost and devastating clinical consequences as a commonly encountered complication.

\section{Conflict of Interest}

Dr. Emami receives grants/research support from $\mathrm{NuVa}$ sive. Dr. Faloon is a consultant for K2M. Dr. Hwang is a consultant for Stryker Spine. None of these are applicable to the current study. For the remaining authors, no potential conflict of interest relevant to this article was reported.

\section{References}

1. Radcliff KE, Kepler CK, Jakoi A, et al. Adjacent segment disease in the lumbar spine following different treatment interventions. Spine J 2013;13:1339-49.

2. Yee TJ, Terman SW, La Marca F, Park P. Comparison of adjacent segment disease after minimally invasive or open transforaminal lumbar interbody fusion. J Clin Neurosci 2014;21:1796-801.

3. Parker SL, Adogwa O, Bydon A, Cheng J, McGirt MJ. Cost-effectiveness of minimally invasive versus open transforaminal lumbar interbody fusion for degenerative spondylolisthesis associated low-back and leg pain over two years. World Neurosurg 2012;78:17884.

4. Radcliff KE, Kepler CK, Maaieh M, et al. What is the rate of lumbar adjacent segment disease after percutaneous versus open fusion? Orthop Surg 2014;6:11820.

5. Epstein NE. Adjacent level disease following lumbar spine surgery: a review. Surg Neurol Int 2015;6(Suppl 24):S591-9.

6. Parker SL, Mendenhall SK, Shau DN, et al. Minimally invasive versus open transforaminal lumbar interbody fusion for degenerative spondylolisthesis: comparative effectiveness and cost-utility analysis. World Neurosurg 2014;82:230-8.

7. Dunn C, Faloon M, Milman E, et al. Accuracy and safety of percutaneous lumbosacral pedicle screw placement using dual-planar intraoperative fluoroscopy. Asian Spine J 2018;12:238-45.

8. Kumar MN, Baklanov A, Chopin D. Correlation between sagittal plane changes and adjacent segment degeneration following lumbar spine fusion. Eur Spine J 2001;10:314-9.

9. Park P, Garton HJ, Gala VC, Hoff JT, McGillicuddy JE. Adjacent segment disease after lumbar or lumbosacral fusion: review of the literature. Spine (Phila Pa 1976) 2004;29:1938-44.

10. Frymoyer JW, Hanley EN Jr, Howe J, Kuhlmann D, Matteri RE. A comparison of radiographic findings in fusion and nonfusion patients ten or more years following lumbar disc surgery. Spine (Phila Pa 1976) 1979;4:435-40.

11. Hilibrand AS, Robbins M. Adjacent segment degeneration and adjacent segment disease: the consequences of spinal fusion? Spine J 2004;4(6 Suppl):190S-4S.

12. Cardoso MJ, Dmitriev AE, Helgeson M, Lehman RA, Kuklo TR, Rosner MK. Does superior-segment facet violation or laminectomy destabilize the adjacent level in lumbar transpedicular fixation? An in vitro human cadaveric assessment. Spine (Phila Pa 1976) 2008:33:2868-73.

13. Battie MC, Videman T, Kaprio J, et al. The Twin Spine Study: contributions to a changing view of disc degeneration. Spine J 2009;9:47-59.

14. Kim DY, Lee SH, Chung SK, Lee HY. Comparison of multifidus muscle atrophy and trunk extension muscle strength: percutaneous versus open pedicle screw fixation. Spine (Phila Pa 1976) 2005;30:123-9.

15. Kim K, Isu T, Sugawara A, Matsumoto R, Isobe M. Comparison of the effect of 3 different approaches to the lumbar spinal canal on postoperative paraspinal muscle damage. Surg Neurol 2008;69:109-13.

16. Park BS, Kwon YJ, Won YS, Shin HC. Minimally Invasive muscle sparing transmuscular microdiscectomy: technique and comparison with conventional subperiosteal microdiscectomy during the early postoperative period. J Korean Neurosurg Soc 2010;48:225-9.

17. Dickerman RD, East JW, Winters K, Tackett J, Hajovsky-Pietla A. Anterior and posterior lumbar interbody fusion with percutaneous pedicle screws: comparison to muscle damage and minimally invasive techniques. Spine (Phila Pa 1976) 2009;34:E9235.

18. Mobbs RJ, Sivabalan P, Li J. Technique, challenges and indications for percutaneous pedicle screw fixation. J Clin Neurosci 2011;18:741-9. 
19. Jones-Quaidoo SM, Djurasovic M, Owens RK 2nd, Carreon LY. Superior articulating facet violation: percutaneous versus open techniques. J Neurosurg Spine 2013;18:593-7.

20. Parker SL, Adamson TE, McGirt MJ, Deshmukh VR. 152; Rate of symptomatic adjacent segment disease after minimally invasive vs open transforaminal lumbar interbody fusion. Neurosurgery 2014;61:210.

21. Seng C, Siddiqui MA, Wong KP, et al. Five-year outcomes of minimally invasive versus open transforaminal lumbar interbody fusion: a matched-pair comparison study. Spine (Phila Pa 1976) 2013;38:204955.
22. Cheh G, Bridwell KH, Lenke LG, et al. Adjacent segment disease followinglumbar/thoracolumbar fusion with pedicle screw instrumentation: a minimum 5-year follow-up. Spine (Phila Pa 1976) 2007;32:2253-7.

23. Ghiselli G, Wang JC, Bhatia NN, Hsu WK, Dawson EG. Adjacent segment degeneration in the lumbar spine. J Bone Joint Surg Am 2004;86:1497-503.

24. Aiki H, Ohwada O, Kobayashi H, et al. Adjacent segment stenosis after lumbar fusion requiring second operation. J Orthop Sci 2005;10:490-5.

25. Zhang C, Berven SH, Fortin M, Weber MH. Adjacent segment degeneration versus disease after lumbar spine fusion for degenerative pathology: a systematic review with meta-analysis of the literature. Clin Spine Surg 2016;29:21-9. 